\title{
Advanced Microwave/Millimeter-Wave Imaging Technology
}

\author{
Zuowei SHEN, Lu YANG, N. C. LUHMANN, Jr., C. W. DOMIER, N. ITO ${ }^{1)}$, Y. KOGI ${ }^{1)}$, Y. LIANG, \\ A. MASE ${ }^{1)}$, H. PARK ${ }^{2)}$, E. SAKATA ${ }^{3)}$, W. TSAI, Z. G. XIA and P. ZHANG \\ Department of Electrical and Computer Engineering, UC Davis, USA \\ 1) Art, Science and Technology Center for Cooperative Research, Kyushu University, Japan \\ ${ }^{2)}$ Princeton Plasma Physics Laboratory, Princeton University, USA \\ ${ }^{3)}$ Kyushu Hitachi Maxell, Ltd, Japan
}

(Received 2 December 2006 / Accepted 11 March 2007)

\begin{abstract}
Millimeter wave technology advances have made possible active and passive millimeter wave imaging for a variety of applications including advanced plasma diagnostics, radio astronomy, atmospheric radiometry, concealed weapon detection, all-weather aircraft landing, contraband goods detection, harbor navigation/surveillance in fog, highway traffic monitoring in fog, helicopter and automotive collision avoidance in fog, and environmental remote sensing data associated with weather, pollution, soil moisture, oil spill detection, and monitoring of forest fires, to name but a few. The primary focus of this paper is on technology advances which have made possible advanced imaging and visualization of magnetohydrodynamic (MHD) fluctuations and microturbulence in fusion plasmas. Topics of particular emphasis include frequency selective surfaces, planar Schottky diode mixer arrays, electronically controlled beam shaping/steering arrays, and high power millimeter wave local oscillator and probe sources.
\end{abstract}

(c) 2007 The Japan Society of Plasma Science and Nuclear Fusion Research

Keywords: plasma diagnostics, electron cyclotron emission imaging (ECEI), microwave imaging reflectometry (MIR), optical system, FSS notch filter, dual dipole antenna array, IF electronics, phased array antenna, MEMs true time delay line

DOI: $10.1585 /$ pfr.2.S1019

\section{Introduction}

Millimeter wave imaging has proven to be a valuable addition to visible, IR, and X-ray imaging systems [1]. Millimeter-wave imaging systems are currently being developed for a wide range of applications, including remote sensing, radio astronomy, environmental measurements, and plasma diagnostics. This paper is focused on those technology advances which have made possible advanced imaging and visualization of magnetohydrodynamic (MHD) fluctuations and microturbulence in fusion plasmas. Topics of particular emphasis include frequency selective surfaces, planar Schottky diode mixer arrays, electronically controlled beam shaping/steering arrays, and high power millimeter wave local oscillator and probe sources.

Millimeter-wave systems are less affected by atmospheric conditions than infrared and visible systems. IR and visible radiation can propagate with little attenuation in clear, dry weather; however, they suffer significant attenuation and scattering when water vapor appears in the form of fog, clouds, and rain. Fortunately, in the millimeterwave regime, there exist propagation "windows" at 35, 94, 140 , and $220 \mathrm{GHz}$, where the attenuation is relatively modest in both clear air and fog. Even taking into account the much higher blackbody radiation at IR and visible fre-

author'se-mail: zshen@ucdavis.edu,luyang@ucdavis.edu, ncluhmann@ucdavis.edu quencies, millimeter waves still provide the strongest signals in fog since millimeter-wave radiation significantly less attenuation under low-visibility conditions than visible or IR radiation. With those advantages, millimeter wave imaging has been applied in a variety of areas including advanced plasma diagnostics, radio astronomy, atmospheric radiometry, concealed weapon detection, allweather aircraft landing, contraband goods detection, helicopter and automotive collision avoidance in fog, harbor navigation/surveillance and highway traffic monitoring in fog, and environmental remote sensing data associated with weather, pollution, soil moisture and oil spill detection [1].

The microwave/millimeter wave (MMW) portion of the electromagnetic spectrum is ideally suited for performing a variety of measurements of magnetic fusion plasma equilibrium parameters as well as their fluctuations. In magnetic plasmas, the conventional technique to measure electron temperature is via a 1-D electron cyclotron emission (ECE) radiometer, and the conventional technique to measure electron density is microwave (non-imaging) radar reflectometry. In a conventional ECE radiometer, a horn antenna receives the ECE radiation at the out board side, which is separated into different frequency bands, each corresponding to a different horizontal location in the plasma (see below for the basic principles). Thus, time-resolved 1-D $T_{\mathrm{e}}$ profiles can be obtained. To obtain 
multi-dimensional temperature profile and fluctuation data, a passive millimeter wave imaging technique, electron cyclotron emission imaging (ECEI) technique, has been developed. Microwave reflectometry first saw use in probing the height of ionospheric plasmas where it was called ionosonde [2]. It is a form of microwave radar that uses the plasma as a reflector and has been widely employed to determine the equilibrium electron density profile [3].

From the outset, microwave reflectometry has also been seen as a tool for helping to understand the relationship between fluctuations and transport by providing high resolution localized measurements of density turbulence in fusion plasmas. Unfortunately, this technique has limited capability in the presence of 2-D fluctuations. Thus, to capture multi-dimensional images of plasma density fluctuations, the microwave imaging reflectrometry (MIR) concept was developed [3].

To resolve the relation between anomalous transport and microturbulence, there is a need for simultaneous $n_{\mathrm{e}}$ and $T_{\mathrm{e}}$ fluctuation measurements. Fortunately, as described below, it has been demonstrated on the TEXTOR tokamak that it is possible to implement a combined ECEI/MIR system to measure both fluctuations simultaneously. These new MMW diagnostics technologies are currently being applied in (or developed for) a number of toroidal devices including TEXTOR [4,5], LHD, NSTX, KSTAR, and EAST to help understand turbulence physics by visualizing plasma temperature and density fluctuations. The paper by Park in this meeting describes recent physics results obtained on TEXTOR using the ECEI system.

In this paper, the MIR and ECEI system concepts and configuration are briefly described. Topics of particular emphasis include technologies such as optical system design, frequency selective surfaces, planar Schottky diode mixer arrays, wide bandwidth IF electronics, electronically controlled beam shaping/steering arrays, and high power millimeter wave local oscillator and probe sources.

\section{MIR and ECEI Concepts}

Microwave reflectometry is a radar technique for the detection of plasma fluctuations from the reflection of microwaves from plasma cut-off surfaces. It has proven to be an extremely useful and sensitive tool for measuring low level density fluctuations in some circumstances; however, this technique has been shown to have limited viability for large amplitude, high $k_{\theta}$ fluctuations, and /or core measurements [6]. The study of the effect of 2-D turbulence on reflectometer measurements led to the development of the Microwave Imaging Reflectometry (MIR) concept. MIR is a technique in which large aperture optics at the plasma edge are used to collect as much of the scattered wave-front as possible and optically focus an image of the cutoff surface onto an array of detectors, thus restoring the integrity of the phase measurement. Figure 1 shows a schematic of the MIR system. The initial off-line laboratory study of the MIR configuration and comparison with a conventional reflectometer arrangement was performed by Munsat [6]. The results showed that the 1-D configuration produces a close match to the reference curve when the distance from the cutoff surface to the image plane is sufficiently small. However, when the distance increases, the 1-D measurement is quite distorted, no longer representing the actual target surface. The MIR system provides an excellent measurement of the wheel surface when it is properly positioned with respect to the target (i.e., within the focal region) $[6,7]$.

In magnetized plasmas, the gyro motion of electrons results in plasma radiation at the electron cyclotron frequency and its harmonics [8]. When the plasma density and temperature are sufficiently high, the plasma becomes optically thick to some harmonics of the electron cyclotron emission (ECE), usually, the first harmonic ordinary mode and the second harmonic extraordinary mode [8,9]. Emission from plasmas of magnetic fusion interest is in the Rayleigh-Jeans regime so that the radiation intensity of optically thick ECE harmonics reaches that of black body radiation. Therefore, the plasma electron temperature and its fluctuations can be determined by measuring the intensity of ECE. In a conventional ECE radiometer, time-resolved 1-D $T_{\mathrm{e}}$ profiles can be obtained. In the 2-D ECEI system, the single antenna in the conventional ECE radiometer is replaced by an array of vertically aligned antennas. Shown in Fig. 2 is a schematic diagram outlining the principle of

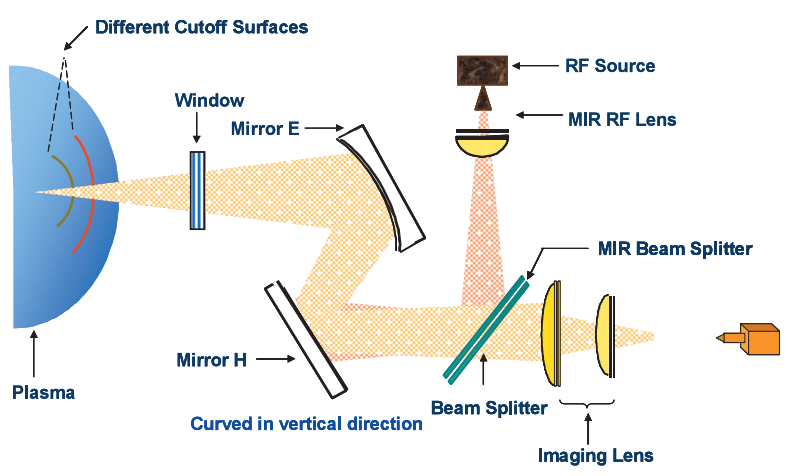

Fig. 1 Schematic representation of MIR system.

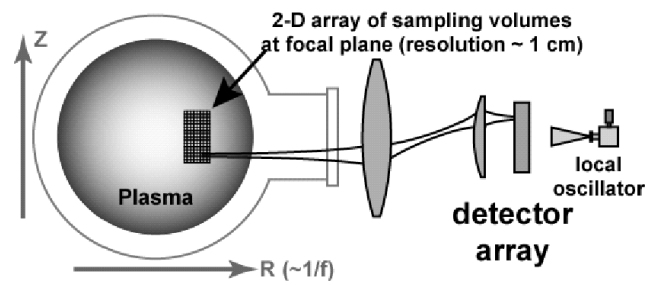

Fig. 2 ECEI system with a quasi-optical 1-D detector array. It is similar to the many vertical layers of a conventional ECE system [5]. 
ECE imaging. ECE radiation is collected and imaged onto a mixer/receiver array comprised of planar antennas with integrated Schottky diodes. The vertically arranged array elements are aligned along the $E$ field (vertical) direction to collect second harmonic X-mode radiation. Large diameter optics image the plasma emission onto the array, enabling each array element to view a distinct plasma chord. The horizontal positions of the sample volumes are determined by the receiver frequencies. Using the one-to-one mapping of ECE frequency to major radius in tokamak plasmas allows the ECEI focal plane to be swept through the plasma by sweeping the receiver frequency of the array, thereby forming 2-D images of the $T_{\mathrm{e}}$ profile [4]. In addition to 2-D measurement capability, ECEI diagnostic systems have excellent spatial resolution $(\sim 1 \mathrm{~cm})$ in both poloidal and toroidal directions. ECEI was first developed and implemented on the TEXT-U tokamak [10,11]. This was followed by an ECEI system on the RTP tokamak [12] and more recently on the TEXTOR tokamak [13]. ECEI systems have now also been developed and installed on mirror machines and stellarators $[14,15]$.

Since both the ECEI and MIR systems require similar collection optics, and the two systems operate in contiguous, but non-overlapping, frequency ranges, it is feasible to combine these two systems by sharing the optical system and window. Consequently, a combined ECEI/MIR system is being developed to simultaneously measure core plasma temperature and density fluctuations in the toroidal machines such as TEXTOR, LHD, and KSTAR. Currently, a combined ECEI and MIR system has been routinely operated on the TEXTOR tokamak. In this system, the primary components of a microwave optical system are shared between ECE and reflectometer subsystems, with each subsystem employing a dedicated high-resolution multichannel detector array. The basic layout was shown in Fig. 3, including a cross-section of the TEXTOR vacuum vessel, the primary focusing mirrors, and the two diagnostic subsystems. Instead of employing a dichroic plate which may lower the sensitivity of both systems, a 50/50 wire grid beam splitter, has been used to separate the two systems.

As illustrated in Fig. 4, the detection portion of ECEI and MIR systems is basically comprised of the imaging optics, heterodyne mixer array, and IF/video electronics. In order to realize the desired broad spatial coverage, all of the elements must support wide band width. The individual technologies are described in the following sections.

\section{Optical Design}

The millimeter wave emission (or reflection) from the plasma exits from a window following which it passes through the optical system and then is imaged onto the MIR and ECEI mixers arrays separately. For ECEI, the goal of the optical design is to achieve the highest possible spatial resolution given constraints such as the port access, need for reasonable dimension optics, and affordable

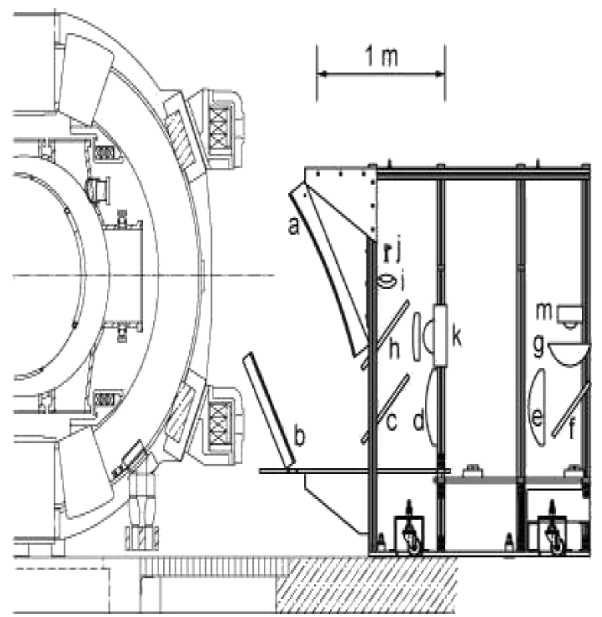

Fig. 3 Detailed schematic of the TEXTOR ECEI/MIR system: (a) Poloidal focusing mirror (b) Toroidal focusing mirror (c) Beam splitter for MIR and ECEI (d) H-plane focusing mirror for ECEI system (e) E-plane focusing mirror for ECEI system (f) Flat mirror (g) Moveable lens for the ECEI system focal depth change (h) beam splitter for MIR source and signal ( $i$ and $j$ ) MIR source beam and collimating lens (k) MIR detection array (m) ECEI detection array [5].

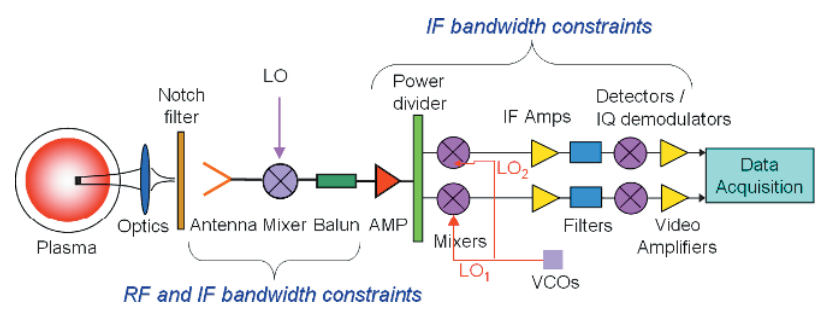

Fig. 4 Constituents of ECEI and MIR detection systems [16].

fabrication cost. For MIR, there is an added complication related to the probing beam. Optics is required to match (both toroidally and poloidally) the probing beam with the curvature of the cutoff surface. The illumination beam and reflected signal are both focused with the optics. Ray tracing and Gaussian propagation analysis are used to provide a one-to-one mapping between the array elements and images and to calculate the focal plane spot size.

For the TEXTOR tokamak, the ECEI and MIR systems share a $42 \mathrm{~cm} \times 42 \mathrm{~cm}$ vacuum window and two large primary focusing vertical aligned cylindrical mirrors as shown in Fig. 5. The mirrors are first designed to tailor the illumination beam wave front to match the cutoff surface. The reflected MIR beam passes through the same window and mirrors, but is separated from the illumination beam by a beam splitter. After passing through the imaging lenses, the reflected signal is collected by the mixer array. The higher frequency $(>110 \mathrm{GHz})$ ECEI signal is separated from the lower frequency $(<90 \mathrm{GHz})$ MIR signal with a dichroic plate as shown and then focused onto the 


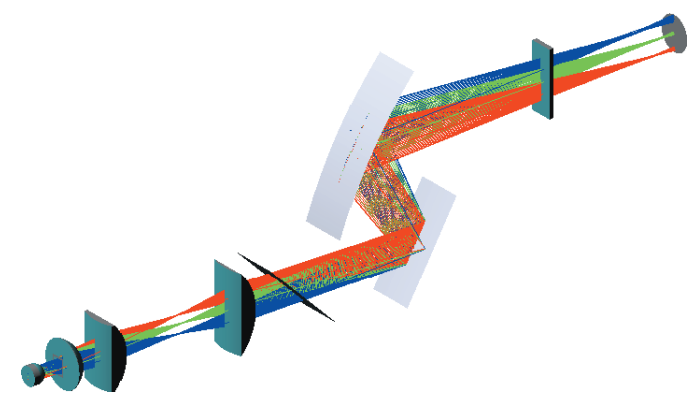

Fig. 5 ECEI ray tracing figure for the TEXTOR tokamak system.

ECEI mixer array using separate imaging optics. Plasma facing cylindrical mirrors are employed instead of lenses because lens surfaces can cause internal reflections which could lead to interference in the case of MIR. The mirrors are comprised of a polystyrene-backed aluminum film bonded to a machined substrate and all lenses are made of high-density polyethylene (HDPE) [17]. The spatial resolution is about $1 \mathrm{~cm}$ at the center channel. Off-axis channels have slightly degraded performance including larger spot size and lower power. Figure 5 illustrates ray-tracing of the optical design in TEXTOR.

For tokamaks with conventional copper coils such as TEXTOR, the diagnostics systems can be mounted on the port covers which are relatively close to the plasma. Consequently, in most cases, the location provides a sufficiently wide field-of-view of the plasma so that there is no need to locate the diagnostics closer to the plasma. However, for devices such as KSTAR the ports are far away from the plasma boundary; consequently, the view coverage is restricted as shown. In KSTAR, to provide a wide field of view, the ECEI/MIR system will therefore be arranged in a single "cassette," which is inserted deep into the long port. The cassette geometry and internal structure place significant constraints on the optical design. Another important issue is that the front end optics experience high heat load due to the long pulse operation with high power. Consequently, the front end optics must be actively cooled and thermal analysis needs to be performed on the optics. From the view point of port-plug and active cooling, the situation of the KSTAR diagnostic is similar to that of the ITER diagnostics.

In KSTAR, the initial imaging optics design is comprised of large mirrors and HDPE lenses as shown in Fig. 6. Large poloidal and toroidal plasma facing stainless steel mirrors are placed within the cassette and shared between the MIR and ECEI subsystems, with each subsystem using high-resolution multi-channel Schottky mixer arrays employing dual dipole antennas. Reflective mirrors are also selected as the focusing optics to avoid spurious reflections from the optics, especially reflections before the microwave signal reaches the plasma in the MIR portion (Fig. 6(a)). The output signals can pass through a rela-

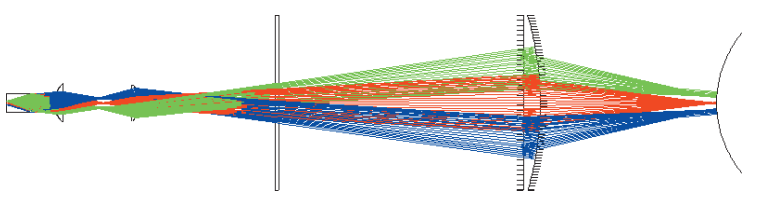

(a)

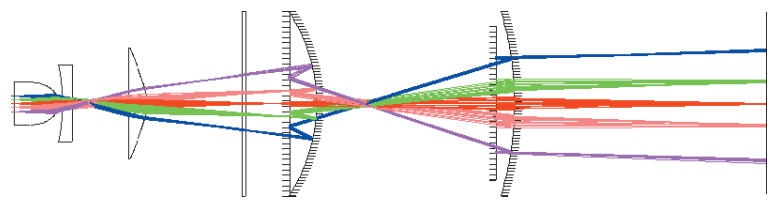

(b)

Fig. 6 KSTAR optical design in the poloidal plane. (a) shows the MIR system with 2 mirrors. (b) shows the ECEI system with 4-mirrors. The two systems share the same front mirrors.

tively small vacuum window. In order to extend the plasma coverage, two additional mirrors are placed within the cassette to extend the plasma coverage (Fig. 6(b)). Optical simulations for 24 channels have been conducted based on a dual dipole antenna array pattern. Different from the TEXTOR ECEI/MIR system [5], the KSTAR MIR and ECEI systems are not separated with a dichroic plate, but rather by utilizing different portions of a mirror. An elliptical lens is used as the antenna substrate lens instead of the hemispherical lens in TEXTOR.

Imaging lenses and the imaging arrays are located in the detection system box which will be aligned with respect to the window on the end plate of a cassette and placed at the KSTAR test cell.

A notch filter (rejection filter) is used in ECEI/MIR systems to protect the mixer arrays from spurious gyrotron heating power. Typical ECRH heating frequencies are $110 \mathrm{GHz}, 140 \mathrm{GHz}$, and $170 \mathrm{GHz}$. Since the heating power is typically several MW, although most of it is absorbed by the plasma, still a small portion will escape and enter the mixer array. The spurious heating power may saturate the detector and decrease its sensitivity; or even worse, burn out the detectors. Because the filter must be mounted between the optical lenses in the imaging system, a frequency selective surface (FSS) is suitable as a thin planar filter and is easy to implement. Frequency selective surfaces consist of an array of periodic metallic patches on a dielectric substrate or a conducting sheet periodically perforated with apertures. Figure 7 shows unit cell structure parameters and a photo of the $140 \mathrm{GHz}$ FSS notch filter. It exhibits total reflection at the resonant frequency. The notch filter is designed with Ansoft Designer, which uses the periodic moment method (PMM). It is fabricated with the novel Electro Fine Forming (EF2) technology by Kyushu Hitachi Maxwell. EF2 allows the circuit to use thinner line widths and gaps as well as sharper edges (compared with standard printed circuit boards as shown in Fig. 8), which 


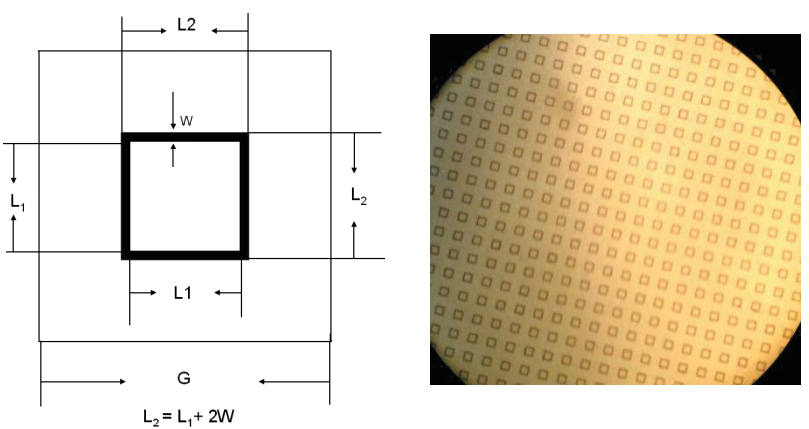

Fig. 7 Left: Square loop unit cell parameters $\mathrm{G}=951 \mu \mathrm{m}, \mathrm{L} 1$ $=320 \mu \mathrm{m}, \mathrm{L} 2=410 \mu \mathrm{m}, \mathrm{w}=45 \mu \mathrm{m}$, right: Photographs of $140 \mathrm{GHz}$ frequency selective surface (FSS) notch filter with square loop structure.
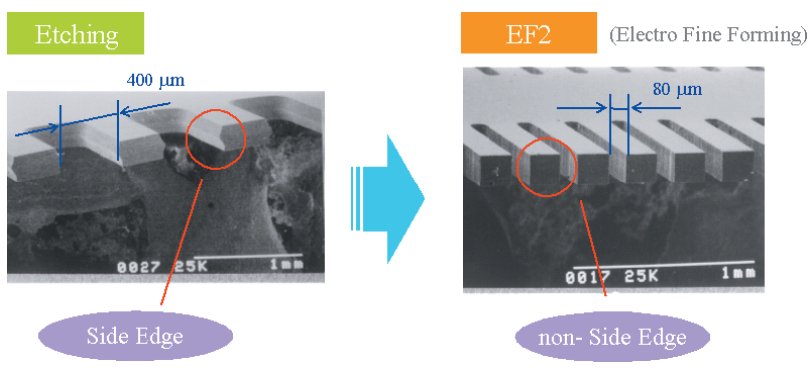

Fig. 8 Line width, gap, and side edges of standard PCB fabrication compared with EF2 technologies.

is required at millimeter wave frequencies.

After comparisons with other unit cell structures such as Jerusalem cross and cross dipole structures, an $8^{\prime \prime} \times 8^{\prime \prime}$ $140 \mathrm{GHz}$ FSS notch filter composed of periodic square loop elements on RO3006 was finally selected to be applied in TEXTOR [18].

The FSS notch filter is measured to have $35 \mathrm{~dB}$ rejection at $140 \mathrm{GHz}$ at normal incidence, and is insensitive to angle of incidence over the range of concern, which is 15 degrees (as shown in Fig. 9). It can provide at least $25 \mathrm{~dB}$ rejection within 15 degrees at $140 \mathrm{GHz}$, with a $Q$ value of 235. In addition to the large rejection, high $Q$, and angle insensitivity, the square loop structure is also easier to fabricate than other structures investigated such as Jerusalem cross and cross dipole structures. The FSS filter can be easily implemented between the lenses and its excellent angle insensitivity allow it to reject most of the spurious heating power even if it is mounted on the lens whose incident beam is uncollimated.

\section{Antenna Arrays}

Quasi-optical planar antenna mixers offer an attractive advantage over wave-guide based mixers at millimeter wave frequencies. They are smaller and lighter than waveguide systems and can be easily produced in large numbers for low cost applications such as millimeter wave imaging

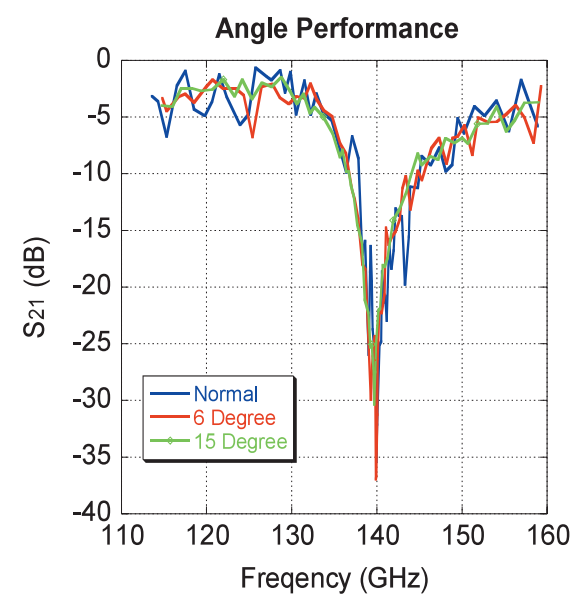

Fig. 9 Transmission performances of the $140 \mathrm{GHz}$ FSS filter at normal incidence, $6^{\circ}$ oblique incidence and $15^{\circ}$ oblique incidence.

systems. The desired planar antenna element for ECEI and MIR imaging array applications should have compact size, be less than one free space wavelength in the vertical direction for high spatial resolution, possess wide RF bandwidth (above 20\%) for wide plasma coverage in the horizontal direction, high directivity $(3 \mathrm{~dB}$ beam width less than 20 degree) for increased receiver sensitivity, low side-lobe levels (less than $10 \mathrm{~dB}$ ) to reduce inter-channel crosstalk, and linear polarization for receiving signals emitted from plasma modes of interest. Dual dipole antenna arrays coupled with elliptical substrate lenses are being investigated for the MIR system on NSTX and the MIR/ECEI system on the KSTAR device. The initial design used an elliptical lens with relative permittivity $\varepsilon_{\mathrm{r}}$ equal to 2.33 , major axis $a=72.8 \mathrm{~mm}$, and minor axis $b=55 \mathrm{~mm}$. The tested dual dipole antenna was fabricated on 20 mils thick Rogers 4350B with $\varepsilon_{\mathrm{r}}$ equal to 3.48 . The antenna far-field patterns are measured in an anechoic chamber. The measured E-plane and $\mathrm{H}$-plane far-field radiation patterns in Q-band and V-band are shown in Fig. 10. The TEXTOR system uses a 16 element antenna array mounted on the hemispherical lens as shown in Fig. 11.

For conventional circular crossection tokamaks, the total magnetic field is approximately a function of only the distance in the radial direction and the center of curvature of the cutoff surface is approximately fixed. Thus, the MIR system can function with only modest changes in focusing over a wide range of plasma conditions. However, the highly shaped, higher $\beta$ plasmas in devices such as NSTX exhibit considerable change in the center of curvature as parameters vary, thereby necessitating the development of electronic beam shaping systems as discussed below. Another recent development thrust has been to move from the use of 1-D detector arrays to the use of 2-D receiver arrays. In this case, the use of multi-frequency illumination together with wideband 2-D MIR antenna arrays makes it 

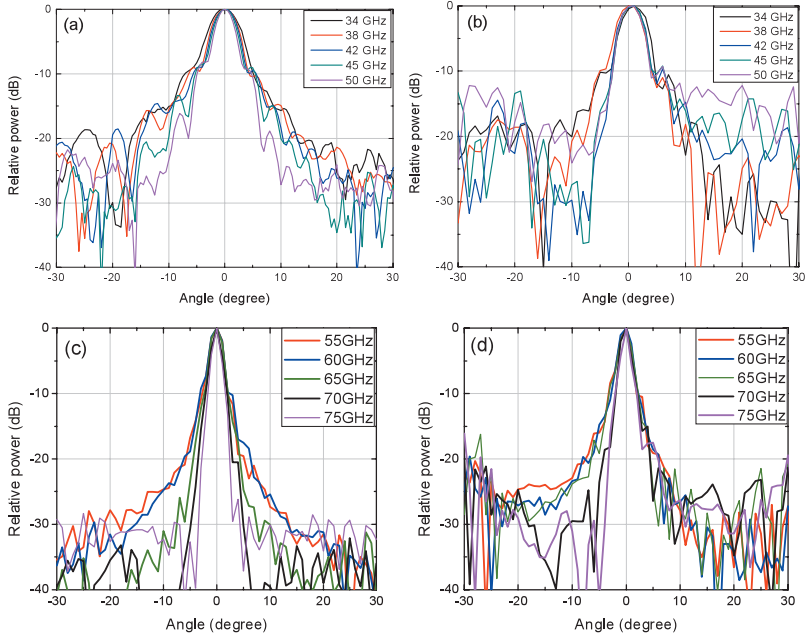

Fig. 10 Measured Q band (a) E-plane, (b) H-plane and V band (c) E-plane and (d) H-plane dual dipole antenna far field radiation patterns on elliptical lens.
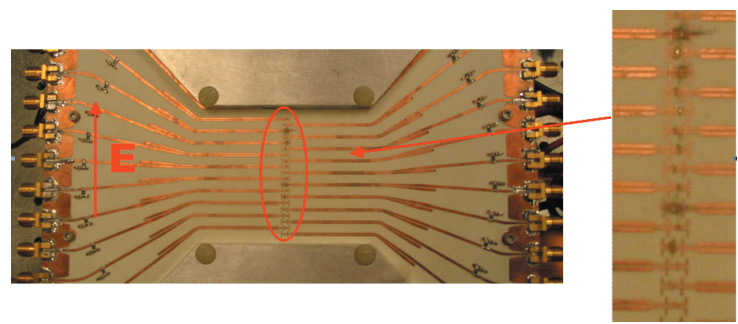

Fig. 11 Heterodyne mixer array for the TEXTOR tokamak ECEI system.

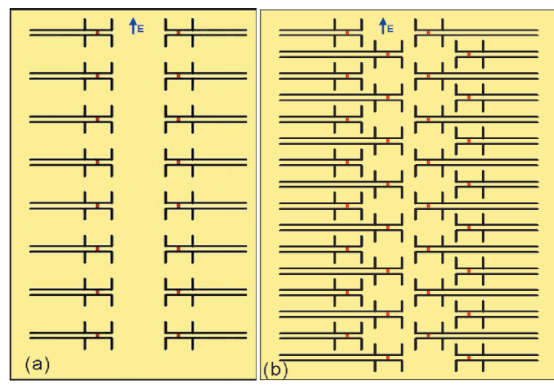

Fig. 12 Schematic layout of $2-D$ (a) $8 \times 2$ and (b) $8 \times 4$ dual dipole antenna arrays.

possible to spatially resolve density fluctuations over an extended 3-D plasma volume. The schematic of a 2-D dual dipole antenna array is shown in Fig. 12.

\section{IF Electronics}

In the NSTX MIR application, a multi-frequency illumination signal will be launched into the plasma. A $44.9 \mathrm{GHz}$ Gunn oscillator will initially serve as the LO source. A $45.0 \mathrm{GHz}$ Gunn oscillator pumps an upconvert-
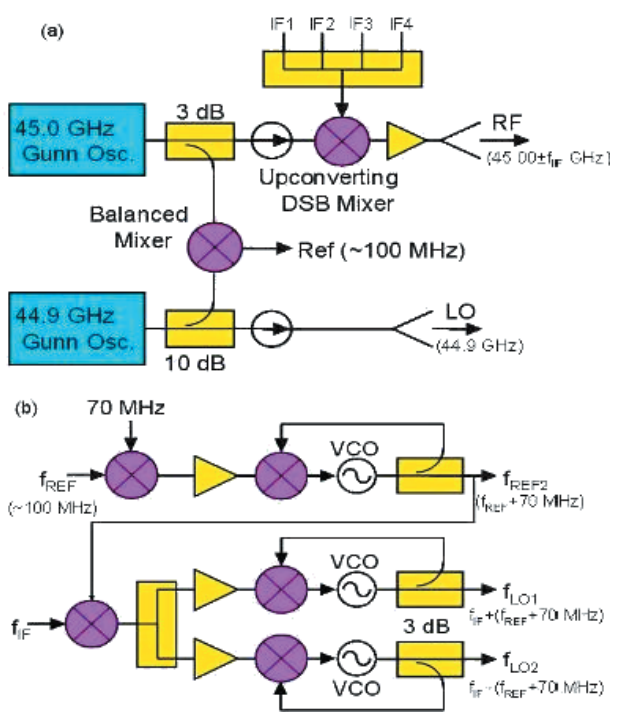

Fig. 13 Approaches of (a) generating multi-frequency illumination signals and (b) LO signals.

ing double sideband (DSB) mixer. Fed with an IF signal of frequency $f_{\mathrm{IF}}$, the DSB mixer generates signals at $45.0 \mathrm{GHz} \pm f_{\mathrm{IF}}$. Fig. 13 (a) shows the block diagram of generating eight illumination signals and the reference signal. The MIR receiver electronics separate out and detect the multiple downconverted MIR signals from each array element. The reflected signals at frequencies $45.0 \mathrm{GHz} \pm f_{\mathrm{IF}}$ are downconverted by the receiver array to frequencies of $f_{\mathrm{IF}} \pm 100 \mathrm{MHz}$. Printed circuit power dividers split the signal from each channel into 8 portions, each of which is down-converted a second time using low cost balanced mixers fed with $0.5-7 \mathrm{GHz}$ LO signals. Fig. 13 (b) illustrates how two of these $8 \mathrm{LO}$ signals can be generated. The resultant down-converted signals are amplified by the IF amplifiers, bandpass filtered and demodulated using the I-Q mixers with a $70 \mathrm{MHz}$ LO signal to generate in-phase and quadrature output signals arising from density fluctuations at a distinct plasma location. This results in an $8 \times 2 \times 8$ or $8 \times 4 \times 8$ mapping of density fluctuations over an extended NSTX plasma volume.

At the ECEI and MIR arrays, the microwave signals are collected by wideband dual-dipole antenna arrays and downconverted to intermediate frequency (IF) signals in the Schottky mixer elements. Subsequently, the signals are amplified by $\sim 35 \mathrm{~dB}$ using low noise preamplifiers, and transmitted to the wideband IF electronics modules, where they are converted to baseband IF signals [19]. In the TEXTOR 128 channel system, the RF circuits equally split each 2.0-8.4 GHz input signal into eight portions with an RF power divider, and each portion is down-converted again using a surface mount mixer with a $2.0-8.4 \mathrm{GHz} \mathrm{LO}$. The second circuit, IF Electronics, rectifies the IF signal from the first circuit to video signals whose amplitude is proportional to the ECE signal strength [19]. 


\section{PAA and MEMS True Time Delay Line Based Beam Shapers}

The fact that the circular-shaped plasma cutoff surfaces of TEXTOR have nearly a common center results in the need to move the horn antenna only over a very short distance to accommodate changes in target plasma conditions. In contrast, for highly shaped plasma machines, such as NSTX, the focal properties of the illumination signal needs to change much more dramatically than those in TEXTOR to match cutoff layers at different locations inside the plasma. Mechanical scanning techniques will be initially employed to vary focal properties between plasma discharges, but such techniques do not provide either the flexibility or the capability of following rapid changes. Consequently, an electronically-controlled beam shaping Phased Array Antenna (PAA) system is under development for application on NSTX. By controlling the illumination signal bandwidth electronically, the PAA system can function like an "artificial lens" and the focal properties of the launching beam can be changed at high speed without mechanically moving the launching antenna. The frequency range of the NSTX MIR instrument is well matched to recently available microwave and millimeter-wave technologies. Specifically, two differ-
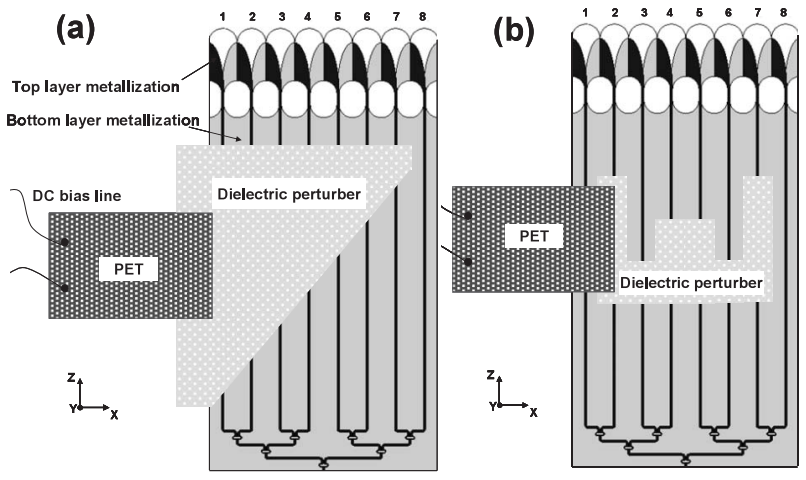

Fig. 14 Structures of Ka-band (a) beam broadening and (b) beam scanning demonstration arrays. ent true time delay technologies to generate the PAA beam shaping are being investigated. First, a Ka-band Piezoelectric Transducer (PET) controlled PAA system using an antipodal elliptically-tapered slot antenna was designed and measured [20]. The $3 \mathrm{~dB}$ beamwidth of the E-plane farfield pattern can be widened by about $10^{\circ}$ and the scanning range is from $-18^{\circ}$ to $+20^{\circ}$ at $30 \mathrm{GHz}$. Figure 14 shows photographs of the Ka-band beam scanning and beam broadening demonstration arrays. Figure 15 shows plots of the perturbed and unperturbed E-plane far field radiation patterns measured at $30 \mathrm{GHz}$.

The second true time delay technology employs microelectromechanical systems (MEMS) extended tuning range varactors periodically loaded on high impedance coplanar waveguide (CPW) transmission lines. A variation in the DC bias of the MEMS varactors results in a change in capacitance, which in turn results in a variation in propagation velocity along the transmission lines and the generation of true time delay for frequencies well below the Bragg cutoff. The usage of MEMS extended range varactor structure eliminated the limitation of pull-in effect of the standard MEMS varactor structure, which gives larger capacitance tuning range and better stability. Figure 16 shows the model of a MEMS extended tuning range varactor and the model employed in the EM simulator. Figure 17 shows an SEM photo of a fabricated Ka-band MEMS extended tuning varactor based true time delay line. The motivation for employing MEMS technology in the true time delay line design is because of the low loss, very high $Q$ at mm-wave frequencies, high power handling capability, and low power consumption together with the relatively
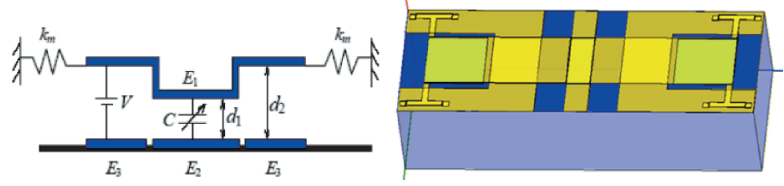

Fig. 16 Modeling of MEMS Extended Tuning Range Varactor.
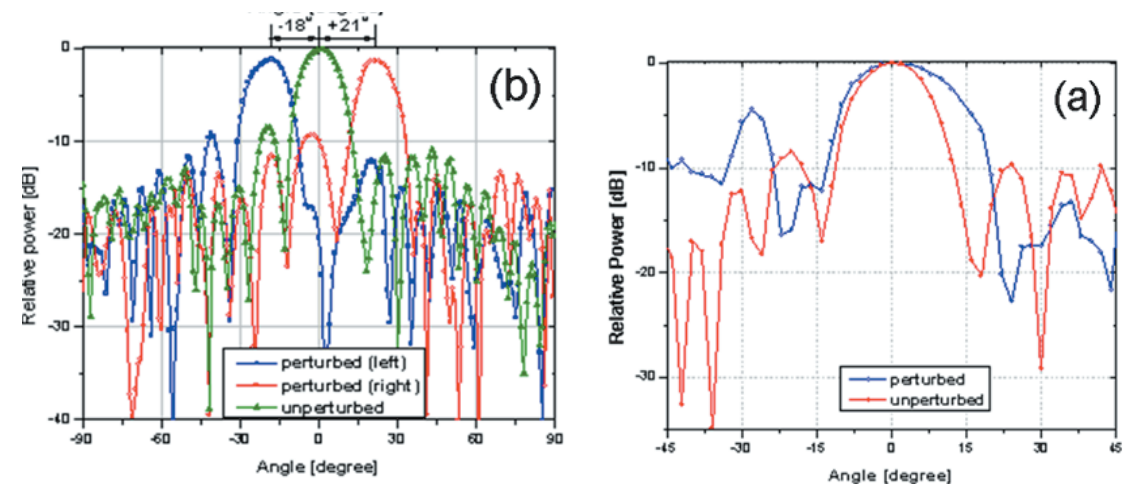

Fig. 15 (a) Beam broadening and (b) beam steering measurement results at $30 \mathrm{GHz}$. 


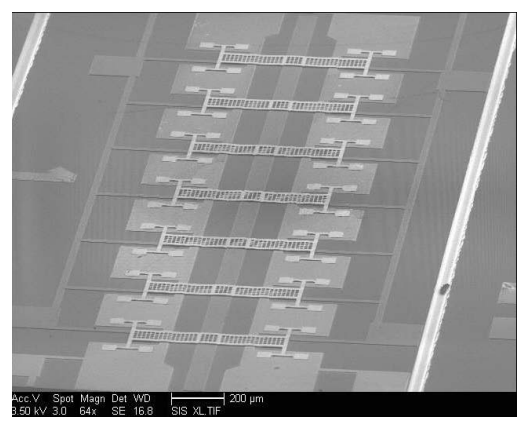

Fig. 17 SEM photo of MEMS varactor true time delay line.

high speed (microseconds).

\section{High Power Millimeter Wave Sources}

The ECEI frequency range for plasma devices currently under investigation extends from approximately $90 \mathrm{GHz}$ to $250 \mathrm{GHz}$. In the case of MIR, the frequency range of interest is approximately $40 \mathrm{GHz}$ to $140 \mathrm{GHz}$. Consequently, relatively high power LO sources are required up to $250 \mathrm{GHz}$ with probe sources extending to $140 \mathrm{GHz}$. Although backward wave oscillators (BWOs) are available in this frequency region, they suffer from a number of limitations including cost, size, limited lifetime, and noise. It is therefore of interest to briefly survey the status of solid state sources. Here, we note that recent advances in millimeter wave technology have resulted in single chip MMIC amplifiers which have provided $427 \mathrm{~mW}$ output at $95 \mathrm{GHz}$ [21], while $2.4 \mathrm{~W}$ in a waveguide combined system [22] has been demonstrated. MMIC amplifiers have been produced at D-Band (110$170 \mathrm{GHz}$ ). Another approach to realize the required local oscillator power for large ECEI arrays is via frequency multiplication. This is predicted to yield $100 \mathrm{~mW}$ at Wband in a MMIC realization [23] and has yielded $5 \mathrm{~W}$ in a quasi-optical frequency tripler grid array [24] at $99 \mathrm{GHz}$. Two recent reviews provide an excellent overview of the current state-of-the-art in spatial power combining $[25,26]$.

\section{Plasma Imaging}

Numerous studies have stressed the need for high resolution imaging diagnostics, which will ultimately permit the visualization of these complicated 2-D and 3-D structures of both electron temperature and density. Examples of recent physics insights obtained through the development of novel imaging diagnostics instrumentation are contained in the review article by Donné [27].

The importance of the ECEI temperature fluctuation visualization capability has been clearly demonstrated through ECEI based studies of the $m=1$ sawtooth instability [28-30]. Here, with the new ECEI "camera," it was demonstrated that during the sawtooth collapse the heat from the center of the plasma flows to the outside via a small perturbation in the magnetic field confining the plasma. The measurements provide a two-dimensional picture of the perturbation of the magnetic field, and this makes it possible to compare the measurements in a very direct way with predictions from various theoretical models. The comparison has led to the conclusion that the quasi-interchange model [31] can be completely discarded, as it cannot describe any of the details seen in the experiment. Two other models that were tested each only can describe a part of the sawteeth evolution. The physical mechanisms unveiled by the 2-D images lead to a new understanding where the reconnection process is identified as a random 3-D local reconnection process with a helical structure. More recent physics studies were conducted on the effect of heating (ECRH) in the suppression of neoclassical tearing modes at the $q=2$ surface by use of data obtained by the 2-D temperature measurements using the ECEI system [32]. Here, the detailed 2D electron temperature information enabled a detailed study of the suppression process and a comparison with theory. This has relevance since suppression of (Neoclassical) Tearing Modes is of great importance for the success of future fusion reactors like ITER. For higher beta plasmas than TEXTOR, mode coupling between sawteeth and tearing modes can occur, processes that can be studied using two ECEI arrays as just one example of future MHD studies made possible with this technology. The time-averaged imaging of microturbulence related temperature fluctuations $\tilde{T}_{e}$ in the plasma core region using intensity interferometric techniques with the ECE diagnostic is described in Ref. [10].

On the reflectometry front, there exist numerous opportunities for imaging including the study of the formation of transport barriers and zonal flows, which often appear to have fine scale structures in profiles, and therefore require high spatial and temporal resolution measurements [33]. Looking ahead to ITER, it has been noted by Vayakis et al. [34] that: "Although robust profiles and some fluctuation information would be available by the present system, it is not able to collect the radiation scattered over a large range of poloidal angles by moderately strong turbulence and as a result, recovery of information on the turbulence amplitude becomes impossible." This argues for the use of reflectometric imaging which has been shown to ameliorate the problem under the proper conditions. One of the limitations of the MIR approach is the difficulty in utilizing large optics in ITER class devices and thus there is interest in other possible amelioration approaches such as synthetically imaging density fluctuations [35]. Here, it should be noted that the synthetic imaging technique is well suited to the array technology discussed in this paper.

\section{Acknowledgments}

This work is supported by the US DOE contract No. DE-FG03-95ER-54295. 
[1] L. Yujiri et al., Microwave Magazine 4 (3) 39 (2003).

[2] K. Bibl, Ann. Geofis. 41, 667 (2000).

[3] E. Mazzucato et al., Rev. Sci. Instrum. 69, 2201 (1998).

[4] B. H. Deng et al., Rev. Sci. Instrum. 72, 301 (2001).

[5] H. Park et al., Rev. Sci. Instrum. 75, 3787 (2004).

[6] T. Munsat et al., Phys. Plasmas 9, 1955 (2002).

[7] T. Munsat et al., Plasma Phys. Control. Fusion 45, 469 (2003).

[8] N.C. Luhmann, Jr. and W.A. Peebles, Rev. Sci. Instrum. 55, 279 (1984)

[9] Neville C. Luhmann, Jr., Instrumentation and Techniques for Plasma Diagnostics (Academic Press, New York, 1979) Ch. 1, pp. 1-65.

[10] B.H. Deng, Phys. Plasmas 8 (5), 2163 (2001).

[11] R.P. Hsia et al., Rev. Sci. Instrum 68, 488 (1997).

[12] B.H. Deng et al., Rev. Sci. Instrum 70, 998 (1999).

[13] B.H. Deng et al., Rev. Sci. Instrum 72, 368 (2001).

[14] A. Mase et al., Fusion Eng. Des. 53, 87 (2001).

[15] A. Mase et al., Rev. Sci. Instrum. 72, 375 (2001).

[16] Z. Shen, C.W. Domier and N.C. Luhmann, Jr., IR-MMWTHz conf proceeding (2006).

[17] T. Munsat et al., Rev. Sci. Instrum. 74, 1426, (2003).

[18] Z. Shen et al., IEEE Antenna \& Propagation Society International Symposium, 4191 (2006).

[19] Lu. Yang, C.W. Domier and N.C. Luhmann, Jr., IEEE Antenna \& Propagation Society International Symposium, 2213 (2006).

[20] C.W. Domier et al., Rev. Sci. Instrum. 77, 10E924 (2006).

[21] Y.C. Chen et al., IEEE Microwave and Guided Wave Lett. 8, 399 (1998).

[22] D.L. Ingram et al., in 2000 IEEE MTT-S International Microwave Symposium Digest, 955 (2000).
[23] E. O'Ciardha, B. Lyons, and S. Lidholm, Int. J. Infrared Millim. Waves 21, 1747 (2001).

[24] H.-X. L. Liu et al., IEEE Electron Device Lett. 14, 329 (1993).

[25] J. Harvey, E.R. Brown, D.B. Rutledge and R.A. York, IEEE Microwave Mag. 1, 48 (2000).

[26] M.P. DeLisio and R.A. York, IEEE Trans. Microwave Theory Tech., Special 50th Anniversary Issue (2001).

[27] A.J.H. Donne, Plasma Phys. Control. Fusion 48 (2006) B483-B496 doi:10.1088/0741-3335/48/12B/S46.

[28] H.K. Park, A.J.H. Donné, N.C. Luhmann, Jr., I.G.J. Classen, C.W. Domier, E. Mazzucato, T. Munsat, M.J. van de Pol, Z. Xia and TEXTOR Team, Phys. Rev. Lett. 96, 195004 (2006).

[29] H.K. Park, N.C. Luhmann, Jr., A.J.H. Donné, I.G.J. Classen, C.W. Domier, E. Mazzucato, T. Munsat, M.J. van de Pol, Z. Xia and TEXTOR Team, Phys. Rev. Lett. 96, 195003 (2006).

[30] H.K. Park, E. Mazzucato, N.C. Luhmann, Jr., C.W. Domier, Z Xia, T. Munsat, A.J.H. Donné, I.G.J. Classen, M.J. van de Pol and TEXTOR Team, Phys. Plasma, 13, 055907 (2006).

[31] J.A. Wesson, Plasma Phys. Control. Fusion 28, 243 (1986).

[32] I.G.J. Classen, E. Westerhof, C.W. Domier, A.J.H. Donné, R.J.E. Jaspers, N.C. Luhmann, Jr., H.K. Park, M.J. van de Pol, G.W. Spakman, M.W. Jakubowski and the TEXTOR Team, Phys. Rev. Lett. 98, 035001 (2007).

[33] N.J. Lopes Cardozo, F.C. Schuller, C.J. Barth et al., Rev. Sci. Instrum. 55, 279 (1994).

[34] G. Vayakis et al., Nucl. Fusion 46, S836 (2006).

[35] G.J. Kramer, R. Nazikian and E.J. Valeo, Plasma Phys. Control Fusion 46, 695 (2004). 